\title{
The Impact of a Multidisciplinary Nursing Model on the Quality of Life of COPD Patients in Remission in the Elderly
}

\author{
Yunlei Gao* \\ Geriatrics Department I, Aerospace Center Hospital, Beijing 100049, China \\ *Corresponding author: Yunlei Gao, gaoyunlei721@163.com
}

Copyright: (C) 2022 Author(s). This is an open-access article distributed under the terms of the Creative Commons Attribution License (CC BY 4.0), permitting distribution and reproduction in any medium, provided the original work is cited.

\begin{abstract}
Objective: To study the effects of multidisciplinary nursing model on self-care ability, lung function, exercise endurance, negative emotion and quality of life of elderly patients with chronic obstructive pulmonary disease (COPD). Methods: 80 patients with COPD hospitalized in the Aerospace Center Hospital from August 2019 to August 2020 were recruited. They were randomly divided into control group and experimental group, with 40 patients in each group. The patients in the control group received routine nursing, and the patients in the experimental group received multidisciplinary nursing intervention on the basis of routine nursing. The nursing observation time of the two groups was 8 weeks. Results: after 8 weeks of nursing intervention, it was found that FEV1 / FVC and 6MWD in the observation group were significantly higher than those in the control group $(\mathrm{P}<0.05)$. There was no significant difference in SAS score and SDS score between the two groups before nursing, but it was significantly lower in the observation group after nursing. The difference between the above data was statistically significant $(\mathrm{P}<0.05)$. The SGRQ score of the observation group was significantly lower than that of the control group $(\mathrm{P}<0.05)$. Conclusion: the application of multidisciplinary nursing model can effectively improve the exercise endurance of elderly patients with COPD, eliminate the negative emotions of patients, and improve the quality of life of patients. The comprehensive application value is significant.
\end{abstract}

Keywords: Multidisciplinary nursing model; Elderly patients with COPD; Quality of life

Online publication: January 19, 2022

\section{Introduction}

Chronic obstructive pulmonary disease (COPD) is a respiratory condition that affects the elderly. Effective nursing interventions must be provided throughout COPD therapy as a type of chronic illness to encourage patients to maintain healthy behavior ${ }^{[1]}$. The interdisciplinary nursing approach launched the most appropriate clinical nursing scheme for patients based on clinical nursing research from many areas. This study aims to include 80 elderly COPD patients to conduct the research, describe the influence on self-care capacity, exercise tolerance, negative emotion, and quality of life in elderly COPD patients using comparative analysis, and investigate its applicability value. The following are the contents.

\section{Data and methods}

\subsection{General information}

From August 2019 to August 2020, 80 elderly patients with COPD were recruited continuously. According to the order of admission, $1 \sim 80$ patients were numbered. 40 patients were randomly assigned as the control 
group to carry out routine nursing. The male to female ratio in the group was 11:9, the age was between $60-78$ years, and the average value was $(65.3 \pm 3.2)$ years; 40 patients were assigned as the observation group, and a multidisciplinary nursing model was carried out. The male to female ratio in the group was $21: 19$, the age ranged from 61 to 78 years, and the average value was $(65.5 \pm 3.3)$ years.

The inclusion criteria ${ }^{[2]}$ : (1) patients over 60 years old; (2) According to "Gold 2020 guidelines," patients with COPD diagnosed by clinical manifestations, pulmonary function examination and imaging examination have no acute exacerbation in recent 12 weeks and are in remission; (3) Patients who have the absolute right to know about this study.

Exclusion criteria: (1) in acute stage of COPD, blood oxygen saturation < 90\%; (2) Pulmonary structural lesions: such as severe pulmonary bullae, pulmonary interstitial diseases; (3) Cardiovascular disease: unstable angina pectoris, recent acute myocardial infarction, severe pulmonary hypertension, severe arrhythmia and cardiac insufficiency; (4) Diseases affecting movement: skeletal and neuromuscular diseases, such as neuromuscular diseases and fractures; (5) Mental illness.

\subsection{Method}

The selected patients received basic nursing work such as walking training, behavior management and drug treatment during the whole treatment process. The nursing time was 8 weeks. The observation group carried out multidisciplinary nursing mode while carrying out routine nursing. The main contents are as follows:

(1) Set up multidisciplinary team (MDT) intervention group, which includes experienced geriatric physicians, geriatric responsible nurses and geriatric specialist nurses Community specialist nurses, nutritionists, rehabilitators, psychologists, etc. all members of the group are jointly responsible for the clinical nursing of patients, and provide professional respiratory disease knowledge training for selected patients, such as issuing COPD health manual, Q\&A, etc. In order to improve the effect of multidisciplinary nursing model, a nursing academic exchange platform was established in the nursing process. All team members of the resources on the platform can learn and use them for clinical nursing of COPD patients ${ }^{[3-5]}$.

(2) During the treatment period, the MDT team conducted a systematic evaluation on the patients, including physical and mental health, negative emotions, exercise endurance, physical health and other aspects. After the evaluation, individual intervention countermeasures were formulated. In terms of physical and mental health: psychological counseling should be carried out for the negative emotions of patients, which should be completed by psychologists and nurses. During the communication process, nurses must keep a mild tone, give patients enough respect and support, establish a sense of trust, master the causes of negative emotions through communication with patients, and formulate targeted treatment plans. For example, in the face of patients who lack the company of their family members, they can contact the family members of the patients to set aside more time to accompany the patients. For example, in the face of patients who lack confidence in treatment, arrange communication meetings for patients who have recovered, and carry out health knowledge training to deepen patients' understanding and understanding of their own condition, so as to firmly believe that they can recover smoothly ${ }^{[6]}$. In terms of exercise endurance: Aiming at the problem of insufficient exercise endurance of patients, carry out upper limbs, lower limbs, rehabilitation treadmill and whole-body walking training, and control the training intensity within the tolerance range of patients to avoid discomfort ${ }^{[7-9]}$. Upper limb training mainly focuses on arm swing within an appropriate range, and lower limb movement mainly focuses on flexion and extension of knee joint and movable ankle joint. Walking training should try to choose the morning with fresh air, maintain aerobic exercise, and stop exercise when patients feel tired. In terms of physical health: respiratory physicians comprehensively evaluate the patient's condition and carry out treatment plans. Cardiopulmonary function training is an important treatment for COPD 
patients. Respiratory muscle training is the main way to improve patients' cardiopulmonary function. The training methods include lip contraction breathing, abdominal breathing, etc. lip contraction breathing refers to breathing with the nose, whistling and exhaling slowly. The respiratory frequency, respiratory depth and degree of lip contraction can be adjusted until the patients do not feel laborious during lip contraction breathing. Abdominal breathing requires patients to take a sitting or standing position, put their right hand on the belly button of the abdomen, and put their left hand on the chest accordingly. When inhaling, expand the abdomen outward to the greatest extent, and when exhaling, compress the abdomen inward to the greatest extent. The respiratory rate is controlled at five to six times per minute, and the duration of abdominal breathing is the best at ten minutes ${ }^{[10]}$.

(3) The MDT group holds regular seminars, and all team members express their opinions, discuss the problems existing in the current patients in their professional fields, and finally formulate intervention measures to provide personalized nursing for patients led by nurses.

(4) Before discharge, MDT team members need to jointly formulate discharge plans suitable for patients, organize patients and their families to publicize nursing methods, and ensure that patients can manage their behavior and participate in rehabilitation training with the help of patients' families and community specialist nurses after discharge ${ }^{[11-12]}$.

(5) After community continuing care is discharged from hospital, members of the group use Internet tools to cooperate with community nurses to return visits to patients. Patients can also rely on the communication platform to consult nursing problems. MDT group members conduct a door-to-door follow-up of selected patients every month, and call all COPD patients in the community to participate in communication activities to master nursing experience and life experience in the communication.

\subsection{Observation indicators}

The first forced expiratory volume (FEV1) / forced vital capacity (FVC) and 6 min walking distance (6MWD) were used as the evaluation indexes of exercise endurance. The negative situation of patients was evaluated by self-rating Anxiety Scale (SAS) and self-rating Depression Scale (SDS) before and after nursing. SGRQ scale was used to evaluate the quality of life of patients, including respiratory symptoms, activity limitation and disease impact. The score was inversely proportional to life. The lower the score, the higher the quality of life. COPD self-efficacy scale (CSES) was used for evaluation, including 5 aspects: negative impact, emotional excitement, physical exertion, weather / environment and behavioral risk factors, with a total of 34 items. Each item has 1-5 points. The higher the score, the stronger the sense of selfefficacy. If the average score is greater than 3 points, it indicates that the ability and self-confidence to promote respiratory management and avoid risk factors are strong ${ }^{[13]}$.

\subsection{Statistical methods}

The obtained data were processed with the help of SPSS18.0, and the measurement data were expressed in the form of mean \pm standard deviation. " $t$ " was used as the detection index, in which $\mathrm{P}<0.05$, the difference was statistically significant.

\section{Results}

\subsection{Comparison of exercise endurance between the two groups}

After 8 weeks of nursing, the FEV1 / FVC value of patients in the observation group was $(84.0 \pm 4.3) \%$, and the FEV1 / FVC value of patients in the control group was $(74.8 \pm 5.2) \%$. There was significant difference between the groups $(\mathrm{t}=8.623, \mathrm{P}=0.000)$; The $6 \mathrm{MWD}$ of patients in the observation group was $(81.5 \pm 6.5) \mathrm{m}$ and that in the control group was $(70.3 \pm 9.2) \mathrm{m}$. there was significant difference between the groups $(\mathrm{t}=6.288, \mathrm{P}=0.000)$. 


\subsection{Comparison of negative emotions between the two groups}

The results before nursing showed that there was no significant difference in negative emotions between the two groups $(\mathrm{P}<0.05)$. After nursing, the SAS scores and SDS scores of patients in the observation group were $(28.5 \pm 12.3),(31.1 \pm 7.8)$ and those in the control group were $(36.3 \pm 9.2),(37.3 \pm 8.6)$. There were significant differences between the two groups $(\mathrm{t}=-3.212, \mathrm{P}=0.002) ;(\mathrm{t}=-3.377, \mathrm{P}=0.001)$.

\subsection{Comparison of quality of life between the two groups}

According to the evaluation results of SGRQ scale of the two groups, the respiratory symptoms, activity limitation, disease impact and total scores of the patients selected in the observation group were significantly lower than those in the control group, and the difference was statistically significant $(\mathrm{P}<0.05)$. See Table 1 for details.

Table 1. Comparison of quality of life between the two groups

\begin{tabular}{lllll}
\hline & Observation group $(\mathbf{n}=\mathbf{4 0})$ & Control group $(\mathbf{n}=\mathbf{4 0})$ & $\mathbf{t}$ & $\mathbf{P}$ \\
\hline Respiratory symptoms & $42.3 \pm 8.4$ & $53.9 \pm 9.8$ & -5.684 & 0.000 \\
\hline Restricted activity & $42.5 \pm 9.3$ & $63.2 \pm 11.6$ & -8.806 & 0.000 \\
\hline Disease impact & $40.4 \pm 6.8$ & $50.8 \pm 10.4$ & -5.293 & 0.000 \\
\hline
\end{tabular}

\subsection{Comparison of self-care ability between the two groups}

There was no significant difference in the scores of self-care ability between the two groups before the intervention $(\mathrm{P}>0.05)$. After the intervention, the scores of patients in the observation group were higher than those before the intervention, and significantly higher than those in the control group $(\mathrm{P}<0.05)$. See Table 2.

Table 2. Comparison of ESCA scores between the two groups ( $x \pm s$, score)

\begin{tabular}{|c|c|c|c|c|c|c|c|c|}
\hline \multirow[t]{2}{*}{ Project } & \multicolumn{2}{|c|}{ Before intervention } & \multirow[t]{2}{*}{$t$} & \multirow[t]{2}{*}{$\mathbf{P}$} & \multicolumn{2}{|c|}{ After intervention } & \multirow[t]{2}{*}{$\mathbf{t}$} & \multirow[t]{2}{*}{$\mathbf{P}$} \\
\hline & $\begin{array}{c}\text { Control } \\
\text { group } \\
(\mathbf{n}=40) \\
\end{array}$ & $\begin{array}{c}\text { Observation } \\
\text { group } \\
(n=40) \\
\end{array}$ & & & $\begin{array}{c}\text { Control group } \\
(n=40)\end{array}$ & $\begin{array}{c}\text { Observation } \\
\text { group }(n=40)\end{array}$ & & \\
\hline Self-care skills & $20.36 \pm 7.32$ & $21.54 \pm 7.89$ & 0.693 & 0.490 & $23.62 \pm 7.45$ & $28.94 \pm 6.58^{*}$ & 3.385 & 0.001 \\
\hline Self-care responsibility & $13.89 \pm 3.65$ & $13.57 \pm 3.44$ & 0.404 & 0.688 & $15.86 \pm 3.71^{*}$ & $18.47 \pm 2.67 *$ & 3.611 & 0.001 \\
\hline Self-concept & $17.14 \pm 5.38$ & $17.94 \pm 5.16$ & 0.679 & 0.499 & $18.55 \pm 4.89$ & $22.64 \pm 5.42 *$ & 3.544 & 0.001 \\
\hline Health knowledge level & $41.87 \pm 9.45$ & $40.86 \pm 9.59$ & 0.474 & 0.637 & $44.76 \pm 8.28$ & $52.31 \pm 9.17 *$ & 3.865 & $<0.001$ \\
\hline $\begin{array}{c}\text { Total score of self-care } \\
\text { ability }\end{array}$ & $96.72 \pm 11.63$ & $95.43 \pm 11.86$ & 0.491 & 0.625 & $104.22 \pm 16.54 *$ & $126.58 \pm 16.37 *$ & 6.077 & $<0.001$ \\
\hline
\end{tabular}

Note: compared with the same group before intervention, $* \mathrm{P}<0.05$

\section{Discussion}

COD has become increasingly common in recent years. Patients will have wheezing and weariness following their sickness, making it difficult for them to live in the best possible condition. Clinical nursing is frequently utilized in COPD treatment to assist and support patient recovery. Passive nursing involvement, on the other hand, may disrupt patients' regular lives, causing worry, impatience, and other emotions, which will subsequently damage the therapeutic result and diminish their quality of life. To address this issue, a 
multi-disciplinary nursing model comprised of multi-disciplinary people was implemented in the clinic, and it was utilized to thoroughly intervene in the nursing work of COPD patients, with positive outcomes. Multidisciplinary nursing mode integrates personnel from multiple disciplines to participate in the clinical nursing of patients and provide personalized nursing services for patients, so as to help patients improve their exercise endurance and negative emotions. Relevant studies have shown that COPD patients will have physiological abnormalities with low FEV1 / FVC value, dyspnea and other symptoms, resulting in decreased enthusiasm for participating in sports training and continuous decline in exercise ability ${ }^{\text {[14] }}$.

Multidisciplinary nursing mode realizes interdisciplinary activities, which is not limited to specialized nursing, provides patients with comprehensive and whole process high-quality nursing services, and improves patients' exercise endurance through aerobic exercise such as walking training. The participation of psychologists has effectively improved various negative emotions of patients, made specialist nurses more purposeful in the process of carrying out nursing services, and improved the nursing effect ${ }^{[15]}$. Selfefficacy is an individual's speculation and judgment on self-ability, and an effective index to predict the health behavior and disease coping ability of patients with chronic diseases ${ }^{[16]}$. The higher the self-efficacy is, the more active it is in daily life, and the better the treatment attitude towards things and emergencies. Improving self-efficacy is helpful for patients to improve their sense of control over the environment and their ability to deal with stress, help patients to maintain self-care habits for a long time and improve their self-care awareness.

Nutritionists and rehabilitation specialists jointly participated in the formulation of a scientific and reasonable diet plan for patients, which is conducive to patients' intake of enough nutrients during treatment, meet their own nutritional needs and maintain good physical condition during treatment. This nursing model makes full use of multi-disciplinary professional skills, realizes all-round and multi angle nursing, and significantly improves the nursing quality and effect. In the study, the improvement of patients' negative emotions under the multidisciplinary nursing model was significantly better than that of routine nursing, and the scores of patients' quality of life were also significantly higher, which fully confirmed the application effect of the multidisciplinary nursing model.

To summarize, the multi-disciplinary nursing model integrates multi-disciplinary professional talents and delivers all-around, multi-angle nursing to patients. It is more personalized than traditional nursing and can successfully improve patients' negative emotions, exercise endurance, and quality of life. The broad application value is significant, and relevant practitioners should refer to it.

\section{Disclosure statement}

The author declares no conflict of interest.

\section{References}

[1] Qi F, Xiao J, 2019, Effects of Multidisciplinary Collaborative Nursing Model on Exercise Endurance and Negative Emotion in Elderly Patients with COPD. Chongqing Medical Journal, 48(03): 154-157.

[2] 2020, Committee. GE. Global Initiative for Chronic Obstructive Lung Disease. Global Strategy for the Diagnosis, Management, and Prevention of Chronic Obstructive Pulmonary Disease 2020 Report. http://www.goldcopd.org

[3] Bi Y, 2019, Effect of Multidisciplinary Collaborative Nursing Model on Exercise Endurance and Negative Emotion of Elderly Patients with COPD. China Health and Nutrition, 017(017): 169-170.

[4] Zhang Y, Wu X, Hu Y, et al., 2019, To Explore the Effects of Multidisciplinary Collaborative Nursing Model on Exercise Endurance, Negative Emotion and Quality of Life of Elderly Patients with Chronic Obstructive Pulmonary Disease (COPD). Electronic Journal of Practical Clinical Nursing, (42). 
[5] Liu K, Zhuang Y, Xu M, 2019, Effects of Multidisciplinary Collaborative Intervention Model on Exercise Endurance, Negative Emotion and Quality of Life in Elderly Patients with Chronic Obstructive Pulmonary Disease. Chinese Journal of Health Psychology, (11).

[6] Cheng H, Yin X, Zi X, 2020, Study on the Correlation between Healthy Psychological Locus of Control and Self-Management in Patients with Stable COPD. Journal of Nursing Education, 35(21): 1984-1988.

[7] Wang Y, 2020, Effect of Cognitive Nursing Intervention on Negative Emotion and Quality of Life of Elderly Patients with COPD. China Health Nutrition, 30(32): 183.

[8] Zhu G, Liu Q, 2016, Effect of Psychological Nursing Intervention on Negative Emotion of COPD Patients. Shanghai Pharmaceutical, 37(21): 51-53.

[9] Yao J, 2018, Effect of Psychological Nursing Intervention on Negative Emotion of COPD Patients. Health Care Guidelines, 000(016): 103.

[10] Enrico C, 2019, Basic Course of Respiratory Rehabilitation, People's Health Publishing House, 1: 111199.

[11] Li H, 2017, Effect of Comprehensive Nursing Intervention on Quality of Life and Negative Emotion of Patients with COPD Complicated with Chronic Respiratory Failure. Chinese Community Physician, (34): 155-157.

[12] Wang J, You Z, Yang C, et al., 2012, Effect of Integrated Medical Care Nursing Intervention on Rapid Rehabilitation of Patients with Esophageal Cancer. Chinese Journal of Modern Nursing, 18(031): 3740-3743.

[13] Zhang Q, Ma H, Zhang S, et al., 2016, Development Status of Continuous Nursing. Nursing research, 30(009): 1028-1030.

[14] Chun-Ju YU, 2016, Hospital FP, The Effect of Nursing Intervention on the Negative Emotion of Patients with Maintenance Hemodialysis, Medical Innovation of China.

[15] Xiaohuan C, Zhihua WU, 2015, Effect of Psychological Nursing Intervention on the Negative Emotion of Patients with Functional Dyspepsia. Clinical Medicine \& Engineering, 2015.

[16] Han Y, Sun Z, Gong Z, et al., 2016, Effects of Individualized Health Management on Self-Efficacy and Compliance Behaviors in Patients with Diabetes Mellitus Complicated with Coronary Heart Disease. Shandong Medicine, 56(38): 65-67. 